\title{
Bijections between Lattice Paths and Plane Partitions
}

\author{
Mateus Alegri ${ }^{1}$, Eduardo Henrique de Mattos Brietzke ${ }^{2}$, José Plínio de Oliveira Santos ${ }^{3 *}$, \\ Robson da Silva ${ }^{4}$ \\ ${ }^{1}$ Departamento de Matemática, UFS, Campus Itabaiana, Itabaiana, Brazil \\ ${ }^{2}$ Instituto de Matemática, UFRGS, Porto Alegre, Brazil \\ ${ }^{3}$ IMECC-UNICAMP, Campinas, Brazil \\ ${ }^{4}$ ICE-UNIFEI, Itajubá, Brazil \\ E-mail: ${ }^{*}$ josepli@ime.unicamp.br
}

Received July 4, 2011; revised August 5, 2011; accepted August 28, 2011

\begin{abstract}
By using lattice paths in the three-dimensional space we obtain bijectively an interpretation for the overpartitions of a positive integer $n$ in terms of a set of plane partitions of $n$. We also exhibit two bijections between unrestricted partitions of $n$ and different subsets of plane partitions of $n$.
\end{abstract}

Keywords: Lattice Paths, Plane Partitions, Partitions, $q$-Analog

\section{Introduction}

In [1], one of us, Santos, in a joint work with Mondek and Ribeiro, introduced a new combinatorial interpretation for partitions in terms of two-line matrices. In that paper a new way of representing, as two-line matrices, unrestricted partitions and several identities from Slater's list [2] including Rogers-Ramanujan Identities, and Lebesgue's Partition Identity is described. In [3] we were able to provide a number of bijective proofs for several identities based on the two-line matrix representation, including a new bijective proof for the Lebesgue Identity, as well as a combinatorial proof for an identity related to three-quadrant Ferrers graphs, given by Andrews (see [4]).

The possibility of associating a partition of $n$ to a two-line matrix and the two-line matrix to a lattice path from the origin to the line $x+y-n$ was mentioned in [1]. This construction can be done in many different ways. Each one of those provides us with a family of polynomials that are, each one, a $q$-analog for the partition function. In order to construct these families it was employed the same idea of counting the lattice path according to the area limited by the path and the $x$-axis, as done by Pólya.

Our main goal in the present paper is to associate a three-line matrix to a path in the 3-dimensional space, in order to build a volume, which is going to correspond to a plane partition. In this way it is possible to construct bijections from certain classes of plane partitions to overpartitions or unrestricted partitions. In some cases mock theta functions appear as the generating functions for these matrices (see [5]).

\section{Background}

In this section we remember a few definitions and state a theorem that is the base for constructing the bijections.

Definition 2.1 A partition of a positive integer $n$ is a collection of positive integers $\lambda_{1} \geq \lambda_{2} \geq \cdots \geq \lambda_{s}$ such that $n=\lambda_{1}+\lambda_{2}+\cdots+\lambda_{s}$. Each $\lambda_{i}$ is called a part of the partition.

Definition 2.2 An overpartition is a partition where the first occurrence of a part can be overlined.

Example 2.1 There are 14 overpartitions of 4 :

$$
\begin{aligned}
& 4, \overline{4}, 3+1, \overline{3}+1,3+\overline{1}, \overline{3}+\overline{1}, 2+2, \overline{2}+2,2+1+1, \overline{2} \\
& +1+1, \overline{2}+\overline{1}+1,2+\overline{1}+1,1+1+1+1, \overline{1}+1+1+1
\end{aligned}
$$

Definition 2.3 A Plane partition $\pi$ is a left-justified array of positive integers $\left(\pi_{i, j}\right)_{i, j \geq 1}$ such that $\pi_{i, j} \geq$ $\max \left\{\pi_{i, j+1}, \pi_{i+1, j}\right\}$. We say that $\pi$ is a plane partition of $n$ if $n=\sum_{i, j \geq 1} \pi_{i, j}$.

Now, if we stack $\pi_{i, j}$ cubes over the position $i, j$ of each part of a plane partition $\pi$, we obtain a geometrical figure in the first octant of the 3-dimensional space, which represents $\pi$ in the same way a Ferrers diagram represents a partition.

Example 2.2 Below (shown in Figures 1 and 2) we have a plane partitions of 43 and its standard geometrical representation. 


$$
\begin{array}{lllll}
4 & 4 & 4 & 3 & 2 \\
4 & 3 & 3 & 2 & 2 \\
2 & 2 & 2 & 1 & \\
2 & 2 & & & \\
1 & & & &
\end{array}
$$

Figure 1. A plane partition.

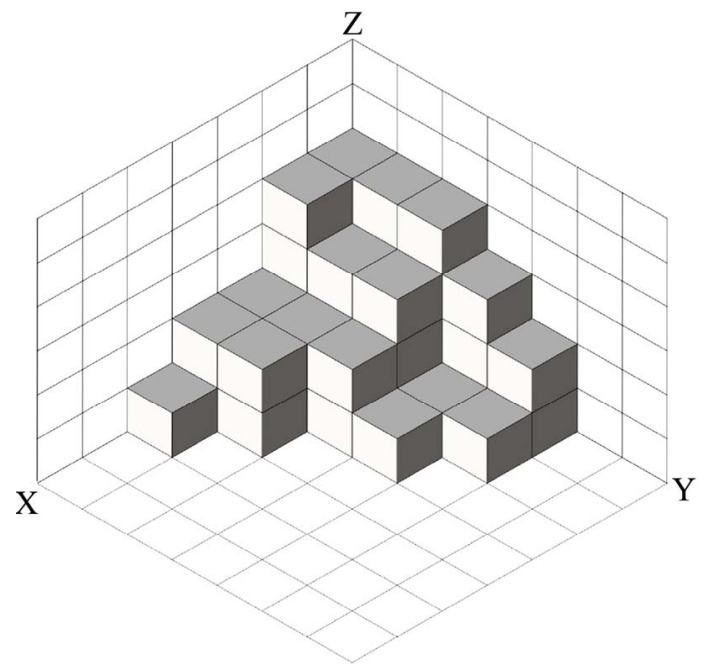

Figure 2. Geometrical representation.

In [6] the following correspondence between threeline matrices and overpartitions is presented.

Theorem 2.1 The number of overpartitions of $n$ is equal to the number of three-line matrices of the form

$$
\left(\begin{array}{lllll}
c_{1} & c_{2} & c_{3} & \cdots & c_{s} \\
d_{1} & d_{2} & d_{3} & \cdots & d_{s} \\
e_{1} & e_{2} & e_{3} & \cdots & e_{s}
\end{array}\right)
$$

with non-negative integer entries satisfying

$$
\begin{aligned}
& e_{t} \in\{0,1\} \\
& c_{s}+e_{s}=1 \\
& e_{t+1}=0 \text { if } d_{t}+e_{t}=e_{t+1} \\
& c_{t}=c_{t+1}+d_{t+1}-i_{t} \\
& \sum c_{t}+\sum d_{t}+\sum e_{t}=n,
\end{aligned}
$$

where $i_{t}=e_{t}$, except when $e_{t}=e_{t+1}=1$ and $c_{t+1}=$, in which case $i_{t}=0$.

The proof of this theorem can be found in [6]. We describe the bijection that follows from the theorem. Given

\begin{tabular}{|c|c|c|c|}
\hline Overpartition & Matrix & Overpartition & Matrix \\
\hline 4 & $\left(\begin{array}{l}1 \\
3 \\
0\end{array}\right)$ & $\overline{4}$ & $\left(\begin{array}{l}0 \\
3 \\
1\end{array}\right)$ \\
\hline $3+1$ & $\left(\begin{array}{ll}1 & 1 \\
2 & 0 \\
0 & 0\end{array}\right)$ & $\overline{3}+1$ & $\left(\begin{array}{ll}0 & 1 \\
2 & 0 \\
1 & 0\end{array}\right)$ \\
\hline $3+\overline{1}$ & $\left(\begin{array}{ll}0 & 0 \\
3 & 0 \\
0 & 1\end{array}\right)$ & $\overline{3}+1$ & $\left(\begin{array}{ll}0 & 0 \\
2 & 0 \\
1 & 1\end{array}\right)$ \\
\hline $2+2$ & $\left(\begin{array}{ll}2 & 1 \\
0 & 1 \\
0 & 0\end{array}\right)$ & $\overline{2}+2$ & $\left(\begin{array}{ll}1 & 1 \\
0 & 1 \\
1 & 0\end{array}\right)$ \\
\hline $2+1+1$ & $\left(\begin{array}{lll}1 & 1 & 1 \\
1 & 0 & 0 \\
0 & 0 & 0\end{array}\right)$ & $\overline{2}+1+1$ & $\left(\begin{array}{lll}0 & 1 & 1 \\
1 & 0 & 0 \\
1 & 0 & 0\end{array}\right)$ \\
\hline $2+\overline{1}+1$ & $\left(\begin{array}{lll}0 & 0 & 1 \\
2 & 0 & 0 \\
0 & 1 & 0\end{array}\right)$ & $\overline{2}+\overline{1}+1$ & $\left(\begin{array}{lll}0 & 0 & 1 \\
1 & 0 & 0 \\
1 & 1 & 0\end{array}\right)$ \\
\hline $1+1+1+1$ & $\left(\begin{array}{llll}1 & 1 & 1 & 1 \\
0 & 0 & 0 & 0 \\
0 & 0 & 0 & 0\end{array}\right)$ & $\overline{1}+1+1+1$ & $\left(\begin{array}{llll}0 & 1 & 1 & 1 \\
0 & 0 & 0 & 0 \\
1 & 0 & 0 & 0\end{array}\right)$ \\
\hline
\end{tabular}
a matrix $A$ of the form (1), the corresponding overpartition $\mu=\mu_{1}+\mu_{2}+\cdots+\mu_{\mathrm{s}}$ is obtained by adding up the entries in each column of $A$. A part $\mu_{t}$ of $\mu$ is marked if and only if $e_{t}=1$. Due to the conditions de- fining matrices of the form (1), in case of more than one column of $A$ add up to the same number, only the left most of these parts is marked.

Example 2.3 In the table below we have the 14 overpartitions of 4 and the corresponding three-line matrices of the form (1).

\section{Bijection between Overpartitions and Plane Partitions via Lattice Path}

In this section we stablish a bijection between the overpartitions of $n$ and certain plane partitions of $n$. In order to do this we describe a possible way to build a volume from a three-line matrix and, then, we associate a plane partition.

Consider, for example, the matrix

$$
\left(\begin{array}{lll}
6 & 3 & 1 \\
4 & 2 & 1 \\
2 & 2 & 0
\end{array}\right)
$$


In order to obtain a solid representing a plane partition we start drawing the lattice path at $(0,1,1)$ moving 6 units in the $x$-direction, 4 units in the $y$-direction, 2 units in $z$-direction, 3 units in the $x$-direction, and so on. In this way we generate a 3-dimensional path. These movements are represented in Figure 3, where the star together with a number near a corner represents a movement in the positive $\mathrm{z}$-direction. When the displacements in both, the $y$ and $z$-directions, are zero, we have a problem to reconstruct the three-line matrix from the lattice path, which can be overcome by the introduction of two different colors.

We need to get from the picture given in Figure 3 a representation for a plane partition in the conventional way, i.e., as a solid in the first octant. It suffices to apply the transformation $x \mapsto-x+\sum_{i=1}^{s} c_{i}+1$, which corresponds to a reflection with respect to the $y z$-plane followed by a translation in the $x$-direction. Figure 4 below shows the corresponding volume.

Note that in the solid shown in Figure $\mathbf{4}$ there is an extra wall added on the back as if at the end of the lattice path we had moved one extra unit in the $x$-direction. This must be done to avoid ambiguity when we reconstruct the three-line matrix from the solid. The plane partition corresponding to this solid is shown below.
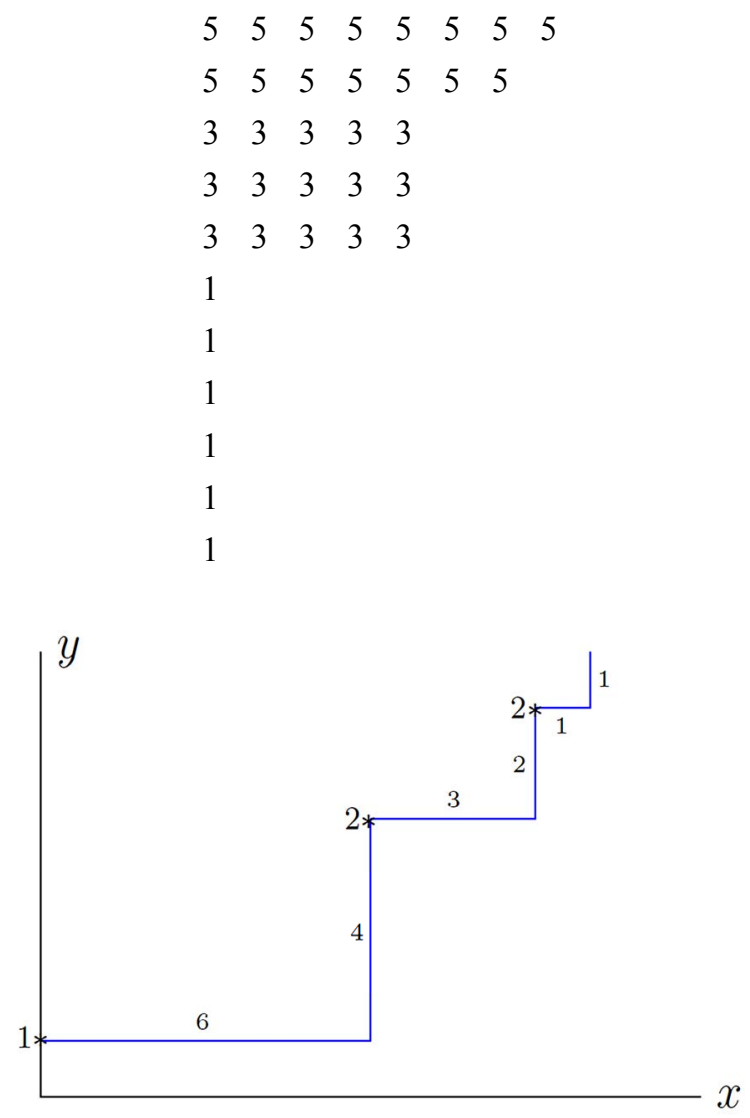

Figure 3. The lattice path.

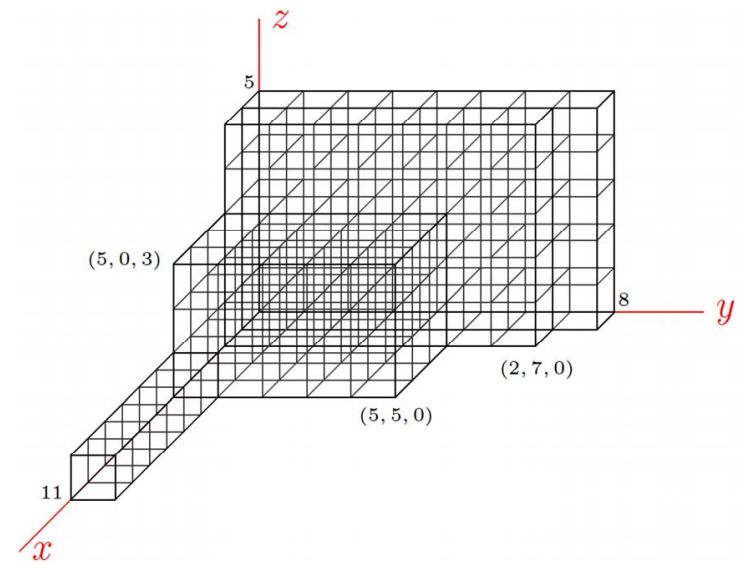

Figure 4. The solid.

As one may see above in Theorem 1, the three-line matrix can have zero entries, in which case we should have to introduce different colors in order to be able to reconstruct the original matrix from the volume. It is important to mention that by the way we are building the lattice path from the matrix the distance from the origin to the end of the path is equal to $n+2$ as shown in Figure 3 (remember we have started at $(0,1,1)$ and, because the extra wall added on the back in Figure 4 the number of layers (11) on the plane partition plus the height of the first layer (5) plus its width (8) must be $n+3 \quad(21+3=24))$.

Now we are going to describe how to solve the problem when there are zero entries so that it will not be necessary to use colors. Consider the overpartition $4+3+$ $\overline{2}+1$ of 10 . By Theorem 1 its three-line matrix representation is

$$
\left(\begin{array}{llll}
3 & 1 & 0 & 1 \\
1 & 2 & 1 & 0 \\
0 & 0 & 1 & 0
\end{array}\right) .
$$

In this case if we draw the lattice path as in Figure $\mathbf{3}$ it would be quite difficult to tell the matrix from which we have started without using colors. One way to solve this problem is to add 1 to each entry of the matrix and then draw the lattice path. In the example above the resulting matrix is

$$
\left(\begin{array}{llll}
4 & 2 & 1 & 2 \\
2 & 3 & 2 & 1 \\
1 & 1 & 2 & 1
\end{array}\right)
$$

and the corresponding figures, as done for matrix (2) are shown in Figure 5-6 below.

Definition 3.1 A plane partition is said to be of type $\mathcal{B}$ if in its geometrical representation the the number of distinct levels that are parallel to each of the planes $x y$, $x z$, and $y z$ is the same. 


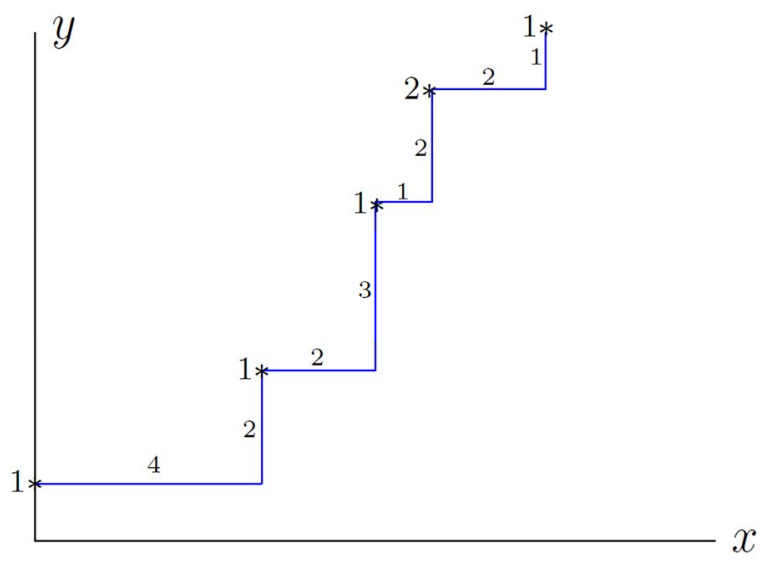

Figure 5. The lattice path.

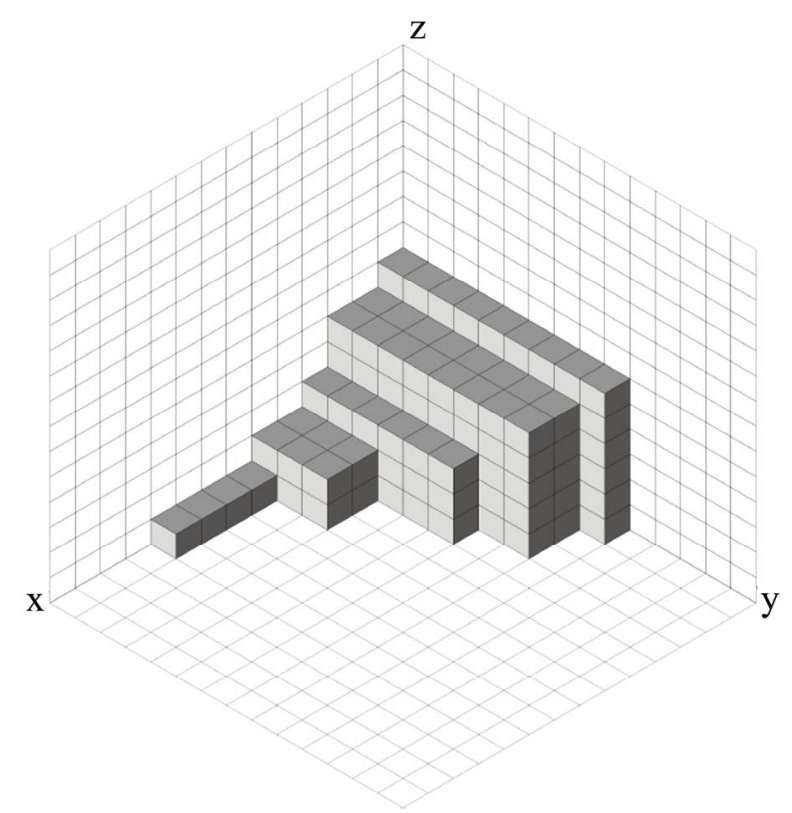

Figure 6. The solid.

Another way to characterize a plane partition of type $\mathcal{B}$ is to say that the number of steps necessary to climb from the $x y$-plane to the top of the solid representing the plane partition is the same as climbing from the $x z$-plane or from the $y z$-plane to the respective top.

From the observations given above we can always move from an overpartition to a 3-line matrix with nonzero entries and, then, drawing the lattice path, get a plane partition of type $\mathcal{B}$. The number of distinct levels that are parallel to each of the planes $x y, x z$, and $y z$ will be one plus the number of columns of the original matrix. This number is precisely the number of blocks (rectangular prisms) that are present in the 3-dimension representation of the plane partition.

Considering the construction above, it is easy to write the matrix with positive entries by looking at the solid representing a plane partition of type $\mathcal{B}$. To get the entries on the third line we have to stand on the plane $z=1$ (remember we have started drawing the lattice path at $(0,1,1))$, climb to the top and set the height of those steps as the entries. For instance, in Figure 6 the steps are, respectively, 1, 1, 2 and 1 . To get the entries on the second line we have to stand on the plane $y=1$ (we have started at $(0,1,1))$, go up to the top and set the height of those steps as the entries. In Figure 6, these steps are respectively, 2, 3, 2 and 1 . As we have made a reflection in the construction of the solids, in order to obtain the entries on the first line we have to stand on the plane $x=$ 1 , climb to the top, count the height of those steps and, then, we set the entries as being these integer in the reverse order. For example, from Figure 6, we have for the first line: 4, 2, 1 and 2 .

The discussion above provides us with a bijection between plane partitions of type $\mathcal{B}$ and those three-line matrices from which by subtracting 1 of each entry we get matrices of the form (1).

Definition 3.2 A plane partition of $n$ is said to be of type $\mathcal{C}$ if it is a plane partition of type $\mathcal{B}$ and the matrix obtained by subtracting 1 from each entry of its corresponding three-line matrix is of the form (1).

We summarize what we have obtained above in the following theorem that gives a complete characterization for the overpartitions in terms of plane partitions.

Theorem 3.1 The number of plane partitions of $n$ of type $\mathcal{C}$ is equal to the the number of overpartitions of $n$.

\section{Two Bijections between Unrestricted Partitions and Plane Partitions}

There is a trivial bijection between unrestricted partitions and plane partitions that can be obtained just by looking a Ferrers diagram as a plane partition with one row. In this section we present two bijections between unrestricted partitions and plane partitions different from the trivial.

In [1] three interpretations as two-line matrices for unrestricted partitions are presented. One of them establishes that:

Theorem 4.1 (Theorem 8 of [1] ) The number of unrestricted partitions of $n$ is equal to the number of matrices of the form

$$
\left(\begin{array}{llll}
c_{1} & c_{2} & \cdots & c_{s} \\
d_{1} & d_{2} & \cdots & d_{s}
\end{array}\right),
$$

where $c_{s}=0, c_{t}=c_{t+1}+d_{t+1}$, and $\sum c_{i}+\sum d_{i}=n$.

The proof of this theorem can be found in [1]. Given a partition $\lambda_{1}+\lambda_{2}+\cdots+\lambda_{s}$ of $n$, we split each part $\lambda_{i}$ into a column starting with $\lambda_{s}$ as the right-most column 
of a matrix of the form (4): $d_{s}=\lambda_{s}, c_{t-1}=c_{t}+d_{t}$ and $d_{t-1}=\lambda_{t-1}-c_{t-1}$. Conversely, given a matrix of the form (4), we obtain a partition of $n$ adding up the entries in each column.

In our next theorem we have an interpretation, as three-line matrices, for unrestricted partitions.

Theorem 4.2 The number of unrestricted partitions of $n$ is equal to the number of three-line matrices of the form

$$
\left(\begin{array}{llll}
c_{1} & c_{2} & \cdots & c_{s} \\
d_{1} & d_{2} & \cdots & d_{s} \\
e_{1} & e_{2} & \cdots & e_{s}
\end{array}\right)
$$

where $c_{s}=d_{s}=c_{s-1}=0, d_{t}=e_{t+1}, e_{s} \geq 1, c_{t}=c_{t+1}+d_{t+1}$, and $\sum c_{i}+\sum d_{i}+\sum e_{i}=n$.

The proof of this theorem is just a consequence of the following notation for the positive integers.

$$
\begin{aligned}
& 1 \mapsto\left(\begin{array}{l}
0 \\
0 \\
1
\end{array}\right) \\
& 2 \mapsto\left(\begin{array}{ll}
0 & 0 \\
1 & 0 \\
0 & 1
\end{array}\right) \\
& 3 \mapsto\left(\begin{array}{lll}
1 & 0 & 0 \\
0 & 1 & 0 \\
0 & 0 & 1
\end{array}\right) \\
& 4 \mapsto\left(\begin{array}{lllll}
1 & 1 & 0 & 0 \\
0 & 0 & 1 & 0 \\
0 & 0 & 0 & 1
\end{array}\right) \\
& 5 \mapsto\left(\begin{array}{lllll}
1 & 1 & 1 & 0 & 0 \\
0 & 0 & 0 & 1 & 0 \\
0 & 0 & 0 & 0 & 1
\end{array}\right) \\
& \vdots \\
& n \mapsto\left(\begin{array}{llllll}
1 & 1 & \cdots & 1 & 0 & 0 \\
0 & 0 & \cdots & 0 & 1 & 0 \\
0 & 0 & \cdots & 0 & 0 & 1
\end{array}\right)
\end{aligned}
$$

We define the sum of two integers in this notation by adding the corresponding matrices in the usual way if they have the same number of columns and, if not, we add to the matrix representing the smaller integer a number of zero columns on the right to have the two matrices with the same number of columns so that we may add in the usual way. For example,

$$
\begin{aligned}
& 5+4 \mapsto\left(\begin{array}{ccccc}
1 & 1 & 1 & 0 & 0 \\
0 & 0 & 0 & 1 & 0 \\
0 & 0 & 0 & 0 & 1
\end{array}\right)+\left(\begin{array}{ccccc}
1 & 1 & 0 & 0 & 0 \\
0 & 0 & 1 & 0 & 0 \\
0 & 0 & 0 & 1 & 0
\end{array}\right) \\
& =\left(\begin{array}{lllll}
2 & 2 & 1 & 0 & 0 \\
0 & 0 & 1 & 1 & 0 \\
0 & 0 & 0 & 1 & 1
\end{array}\right),
\end{aligned}
$$

and

$$
9+8+8+5+4+4 \mapsto\left(\begin{array}{ccccccccc}
6 & 6 & 4 & 3 & 3 & 3 & 1 & 0 & 0 \\
0 & 0 & 2 & 1 & 0 & 0 & 2 & 1 & 0 \\
0 & 0 & 0 & 2 & 1 & 0 & 0 & 2 & 1
\end{array}\right) .
$$

With this three-line matrix representation for unrestricted partitions and exactly the same construction described in the previous section for overpartitions we can state a nontrivial characterization for unrestricted partitions as plane partitions.

Definition 4.1 A plane partition of $n$ is said to be of type $\mathcal{D}$ if it is a plane partition of type $\mathcal{B}$ and the matrix obtained by subtracting 1 from each entry of its corresponding three-line matrix is of the form (5).

Theorem 4.3 The number of plane partitions of $n$ of type $\mathcal{D}$ is equal to the the number of unrestricted partitions of $n$.

Consider, for example, the partition $5+4+2+2+1$. Then

$$
5+4+2+2+1 \mapsto\left(\begin{array}{ccccc}
2 & 2 & 1 & 0 & 0 \\
2 & 0 & 1 & 1 & 0 \\
1 & 2 & 0 & 1 & 1
\end{array}\right) \mapsto\left(\begin{array}{lllll}
3 & 3 & 2 & 1 & 1 \\
3 & 1 & 2 & 2 & 1 \\
2 & 3 & 1 & 2 & 2
\end{array}\right) .
$$

Now we can obtain the plane partition associated to $5+4+2+2+1$ by drawing the lattice path (Figure 7) and constructing the solid (Figure 8) as before:

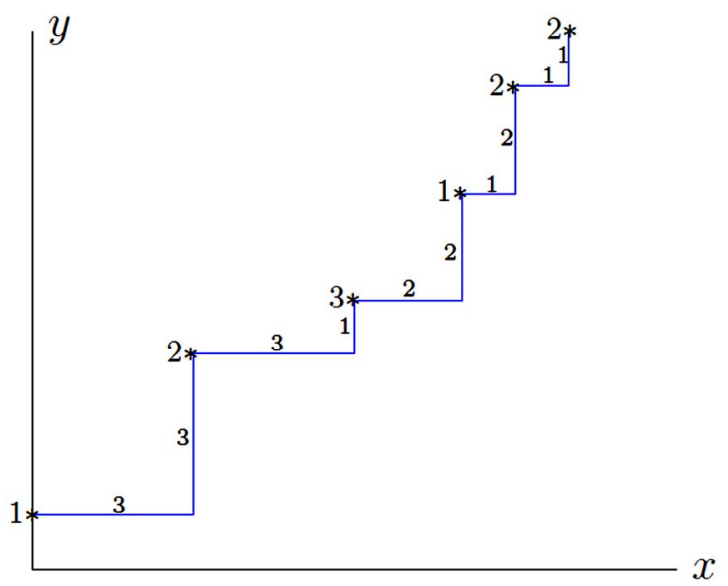

Figure 7. The lattice path. 


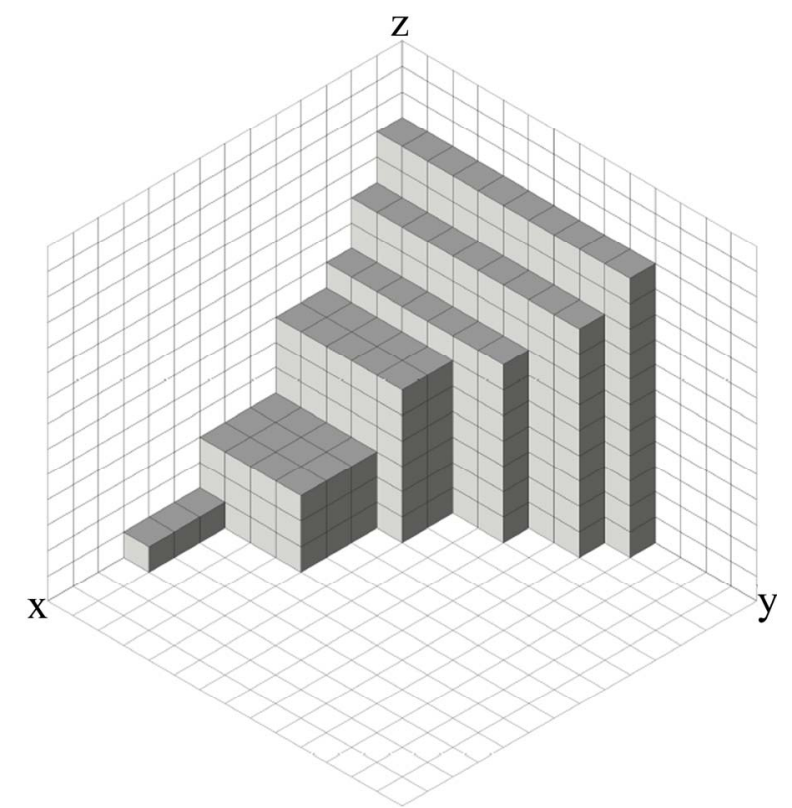

Figure 8. The plane partition.

As an extra example consider the partition $6+3+$ $3+2$. Then

$$
\begin{aligned}
& 6+3+3+2 \mapsto\left(\begin{array}{llllll}
3 & 1 & 1 & 1 & 0 & 0 \\
1 & 2 & 0 & 0 & 1 & 0 \\
0 & 1 & 2 & 0 & 1 & 1
\end{array}\right) \\
& \mapsto\left(\begin{array}{llllll}
4 & 2 & 2 & 2 & 1 & 1 \\
2 & 3 & 1 & 1 & 2 & 1 \\
1 & 2 & 3 & 1 & 1 & 2
\end{array}\right)
\end{aligned}
$$

and the lattice path and the corresponding plane partition associated to $6+3+3+2$ are shown in Figures 9 and 10.

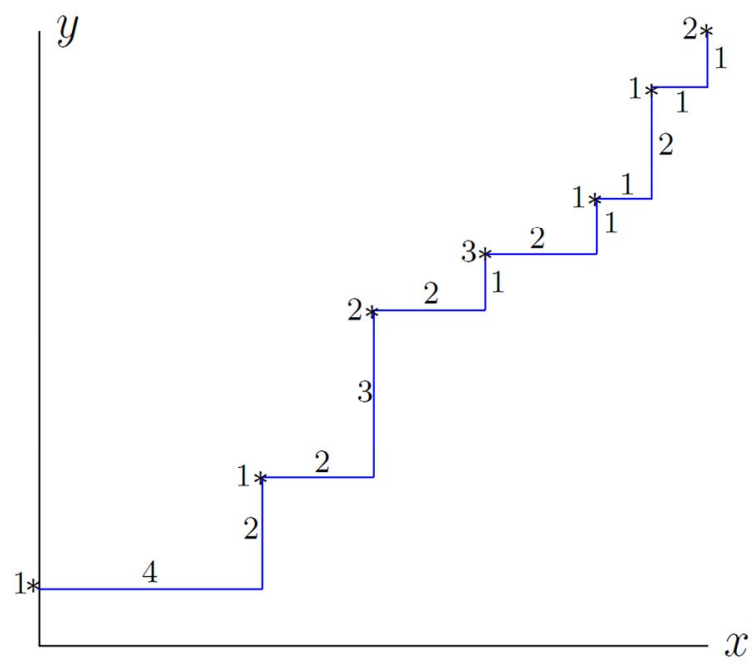

Figure 9. The lattice path.

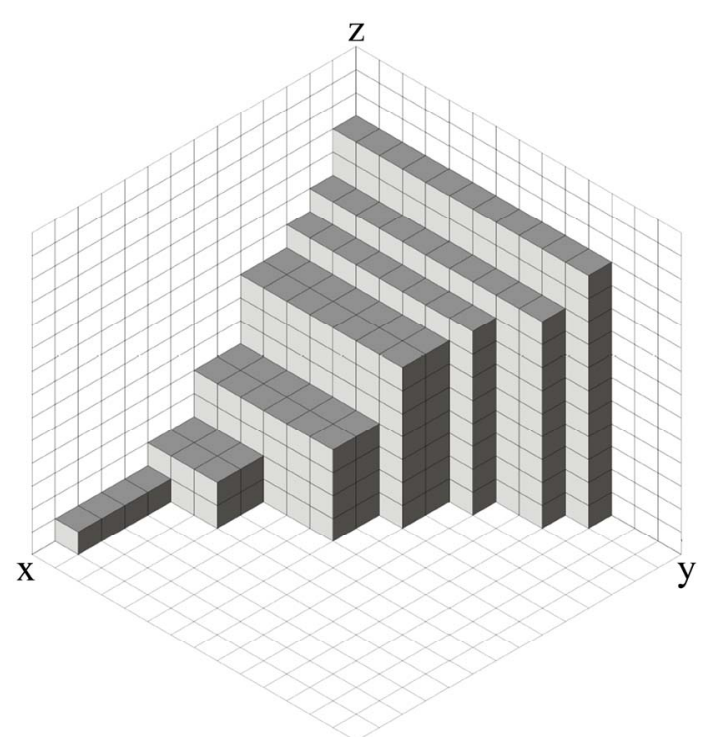

Figure 10. The plane partition.

Our final result is another representation for unrestricted partitions as plane partitions different from the one just described. To do this we use the result given in Theorem 4.2. From the restrictions defining a matrix of the form (5) it is easy to see that the third line, without $e_{1}$, is equal to the second one (just shifted one position to the right). Now we can associate to each one of these threeline matrices a two-line matrix by adding together the elements $d_{t}$ and $e_{t+1}$ that are equal. This is equivalent to multiply the second line by 2 . We add the element $e_{1}$ to the first entry $c_{1}$ on the first line. We observe that, in general, the number of columns in the two-line matrix obtained is reduced by one. Te only case in which this number is not reduced is when the three-line matrix has only one column. In this case we define the corresponding two-line matrix by:

$$
\left(\begin{array}{l}
0 \\
0 \\
n
\end{array}\right) \mapsto\left\{\begin{array}{l}
\left(\begin{array}{l}
1 \\
n-1
\end{array}\right), \text { if } n \text { is even } \\
\left(\begin{array}{l}
0 \\
n
\end{array}\right), \text { if } n \text { is odd. }
\end{array}\right.
$$

To illustrate the bijection just defined we list below two examples

$$
\begin{aligned}
& \left(\begin{array}{lllll}
2 & 2 & 1 & 0 & 0 \\
2 & 0 & 1 & 1 & 0 \\
1 & 2 & 0 & 1 & 1
\end{array}\right) \mapsto\left(\begin{array}{llll}
3 & 2 & 1 & 0 \\
4 & 0 & 2 & 2
\end{array}\right) \\
& \left(\begin{array}{llllll}
3 & 1 & 1 & 1 & 0 & 0 \\
1 & 2 & 0 & 0 & 1 & 0 \\
0 & 1 & 2 & 0 & 0 & 1
\end{array}\right) \mapsto\left(\begin{array}{lllll}
3 & 1 & 1 & 1 & 0 \\
2 & 4 & 0 & 0 & 2
\end{array}\right)
\end{aligned}
$$


From this bijection we have the following theorem.

Theorem 4.4 The number of unrestricted partitions of $n$ is equal to the number of two-line matrices of the form

$$
\left(\begin{array}{cccc}
c_{1} & c_{2} & \cdots & c_{s} \\
d_{1} & d_{2} & \cdots & d_{s}
\end{array}\right)
$$

where

- for $s>1, c_{s}=0, d_{t}$ is even with , $c_{1} \geq c_{2}+\frac{d_{2}}{2}$,

$$
c_{t}=c_{t+1}+\frac{d_{t+1}}{2} \text { for } t>1 \text {; }
$$

- for $s=1$ and $n$ even, $c_{1}=1$ and $d_{1}=n-1$;

- for $s=1$ and $n$ odd, $c_{1}=0$ and $d_{1}=n$;

- and $\sum c_{i}+\sum d_{i}=n$.

In order to get, for an integer $n$, a complete description for another subset of plane partitions having the same cardinality as the number of unrestricted partitions of $n$ we do the following.

When we moved from a matrix of the form (5) to one like (6) we have added the entries $e_{1}$ and $c_{1}$ of (5) to get the entry $c_{1}$ of (6). Now we consider the two-line matrix without this addition and use this matrix to draw a lattice path, as we did before, by first adding one to each entry. Observing that this lattice path is entirely on the plane $z=1$ we use the element $e_{1}$ to move up this lattice path to the plane $z=1+e_{1}$. This operation is illustrated below.

$$
\begin{aligned}
& 5+4+2+2+1 \mapsto\left(\begin{array}{ccccc}
2 & 2 & 1 & 0 & 0 \\
2 & 0 & 1 & 1 & 0 \\
1 & 2 & 0 & 1 & 1
\end{array}\right) \\
& \mapsto\left(\begin{array}{llll}
2 & 2 & 1 & 0 \\
4 & 0 & 2 & 2
\end{array}\right) \mapsto\left(\begin{array}{llll}
3 & 3 & 2 & 1 \\
5 & 1 & 3 & 2
\end{array}\right)
\end{aligned}
$$

The corresponding lattice path and plane partition are shown in Figures 11 and 12.

Definition 4.2 A plane partition is said to be of type $E$ if the number of faces that are parallel to the $x z$-plane is equal to the number of faces that are parallel to the $y z$-plane and there is only one face parallel and above the xy-plane.

Definition 4.3 A plane partition of type $\mathcal{F}$ is a plane partition of type $E$ such that the matrix obtained from its corresponding two-line matrix with nonzero entries after subtracting 1 from each entry and adding $e_{1}$ to $c_{1}$ is of the form (6).

Figure 10 gives an example of a plane partition of type $\mathcal{F}$. Observe that there is only one face (on the plane $z=1+e_{1}$ ) parallel and above the $x y$-plane. With this definitions and the discussion above we now state our last theorem.

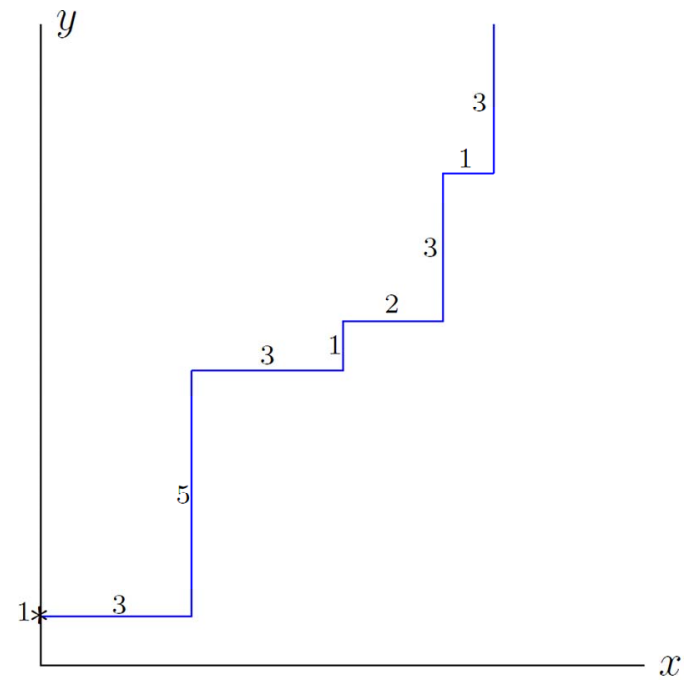

Figure 11. The lattice path.

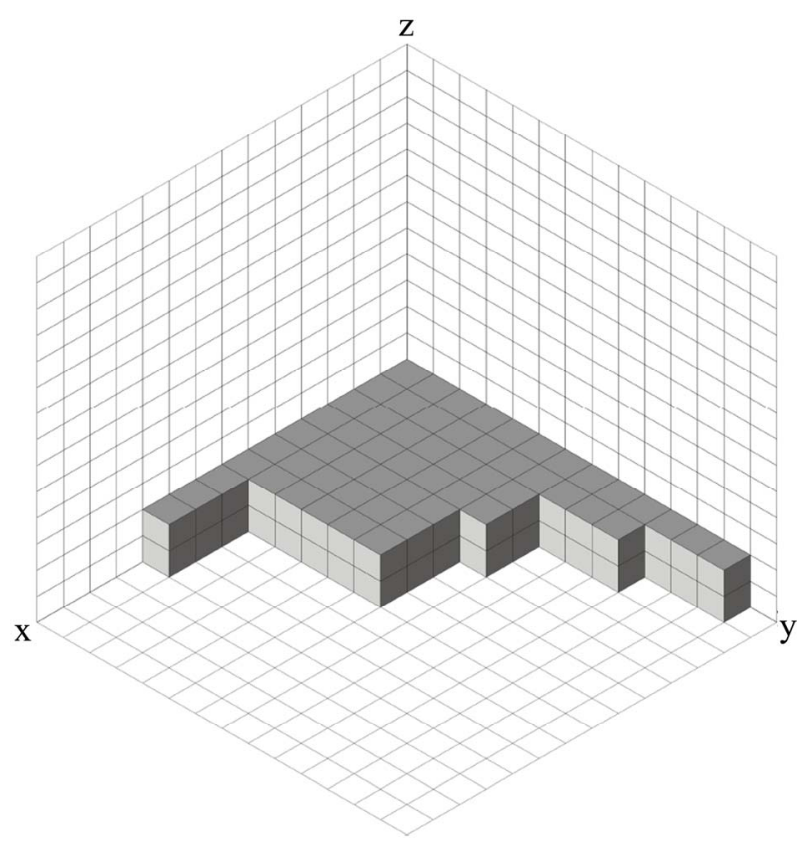

Figure 12. The plane partition.

Theorem 4.5 The number of plane partitions of $n$ of type $\mathcal{F}$ is equal to the number of unrestricted partitions of $n$.

\section{References}

[1] P. Mondek, A. C. Ribeiro and J. P. O. Santos, "New Two-Line Arrays Representing Partitions," Annals of Combinatorics, Vol. 15, No. 2, 2011, pp. 341-354. doi:10.1007/s00026-011-0099-0

[2] L. J. Slater, "Further Identities of the Rogers-Ramanujan Type," Proceedings of the London Mathematical Society, Vol. 54, No. 2, 1952, pp. 147-167. 
doi:10.1112/plms/s2-54.2.147

[3] E. H. M. Brietzke, J. P. O. Santos and R. Silva, "Bijective Proofs Using Two-Line Matrix Representations for Partitions," The Ramanujam Journal, Vol. 23, 2010, pp. 265295.doi:10.1007/s11139-009-9207-8

[4] G. E. Andrews, "Three-Quadrant Ferrers Graphs," Indian Journal of Mathematics, Vol. 42, 2000, pp. 1-7.
[5] E. H. M. Brietzke, J. P. O. Santos and R. Silva, "Combinatorial Interpretations as Two-Line Array for the Mock Theta Functions," Bulletin Brazilian Mathematical Society, Vol. 44, 2013, pp. 233-253.

[6] M. Alegri, "Interpretações para Identidades Envolvendo Sobrepartições e Partições Planas," Ph.D. Thesis, IME CC-Universidade Estadual de Campinas, Campinas-SP, 2010 . 